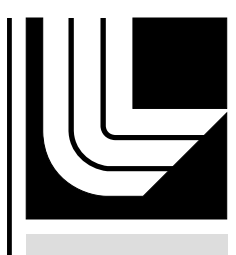

LAW RENCE LIVERMORE N A T IO N A L LABORATORY

EDC-37 Deflagration Rates at Elevated Pressures

J. L. Maienschein, J. G. Koerner

February 4, 2008 
This document was prepared as an account of work sponsored by an agency of the United States government. Neither the United States government nor Lawrence Livermore National Security, LLC, nor any of their employees makes any warranty, expressed or implied, or assumes any legal liability or responsibility for the accuracy, completeness, or usefulness of any information, apparatus, product, or process disclosed, or represents that its use would not infringe privately owned rights. Reference herein to any specific commercial product, process, or service by trade name, trademark, manufacturer, or otherwise does not necessarily constitute or imply its endorsement, recommendation, or favoring by the United States government or Lawrence Livermore National Security, LLC. The views and opinions of authors expressed herein do not necessarily state or reflect those of the United States government or Lawrence Livermore National Security, LLC, and shall not be used for advertising or product endorsement purposes.

This work performed under the auspices of the U.S. Department of Energy by Lawrence Livermore National Laboratory under Contract DE-AC52-07NA27344. 


\title{
EDC-37 DEFLAGRATION RATES AT ELEVATED PRESSURES
}

\author{
Jon Maienschein, Jake Koerner \\ Lawrence Livermore National Laboratory Energetic Materials Center \\ Livermore, CA USA
}

\section{INTRODUCTION}

We report deflagration rates on EDC-37 at high pressures. Experiments are conducted using the Lawrence Livermore National Laboratory High Pressure Strand Burner (HPSB) apparatus. The HPSB contains a deflagrating sample in a small volume, high pressure chamber. The sample consists of nine, $6.35 \mathrm{~mm}$ diameter, $6.35 \mathrm{~mm}$ length cylinders stacked on end, with burn wires placed between cylinders. Sample deflagration is limited to the cross-sectional surface of the cylinder by coating the cylindrical surface of the tower with Halthane 88-2 epoxy. Sample deflagration is initiated on one end of the tower by a $\mathrm{B} / \mathrm{KNO}_{3}$ and $\mathrm{HNS}$ igniter train. Simultaneous temporal pressure history and burn front time of arrival measurements yield the laminar deflagration rate for a range of pressures and provide insight into deflagration uniformity. These measurements are one indicator of overall thermal explosion violence. Specific details of the experiment and the apparatus can be found in the literature. ${ }^{1}$

\section{RESULTS AND DISCUSSION}

EDC-37 powder from LLNL Lot \# C-425 was uniaxially pressed in a cylindrical pressing die at $45^{\circ} \mathrm{C}$ to form cylindrical pellets used in an experiment. Each pellet was pressed at $200 \mathrm{MPa}$ $(30,000 \mathrm{psi})$ for three, five minute dwells. The tacky nature of the sample made storing finished pellets in individual glass vials desirable. Samples were pressed to a density of approximately $1.825 \mathrm{~g} / \mathrm{cm}^{3}+/-0.001 \mathrm{~g} / \mathrm{cm}^{3}$, which is approximately $99.2 \%$ of the sample's theoretical maximum density of $1.84 \mathrm{~g} / \mathrm{cm}^{3}$.

Typical data from a run conducted in 2006 is shown in Figure 1 below. Burn wires report evenly and in order and the pressure increases in a smooth fashion, although more rapidly near the end of the run.

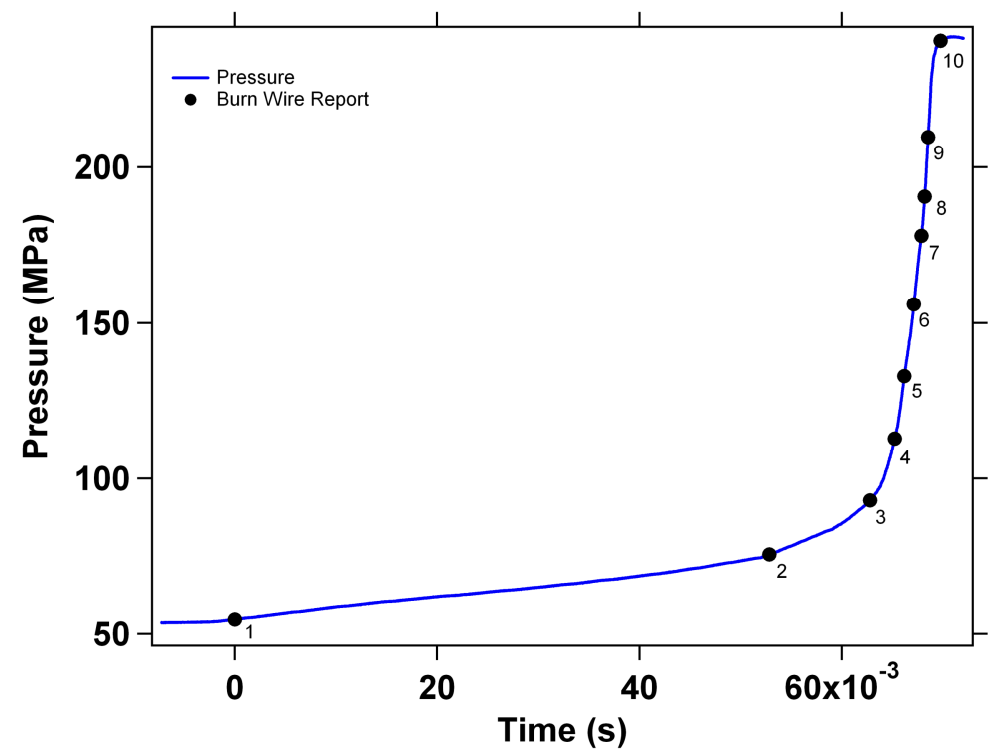

FIGURE 1. EDC-37 temporal pressure history and burn front time-of-arrival for one run.

This work performed under the auspices of the U.S. Department of Energy by Lawrence Livermore National Laboratory under Contract DE-AC52-07NA27344. 
During runs conducted in 2004 , extremely elevated deflagration rates were occasionally observed. We conjecture that the increase in deflagration rate was caused by an unfavorable interaction of explosive and epoxy at the explosive/epoxy interface and allowed wicking of the flame down that interface. Further experimentation revealed the elevated deflagration rates were reduced if the time of explosive/epoxy interaction was reduced. Consequently, for runs completed in 2006, special care was taken to ensure that experiments were conducted soon after the cylindrical surface of the tower was coated with epoxy. Usually, the time lapse was less than two days.

Several runs conducted over a wide range of pressures yield the deflagration rate data shown below in Figure 2. All runs were conducted at ambient temperature, $21^{\circ} \mathrm{C}\left(70^{\circ} \mathrm{F}\right)$.

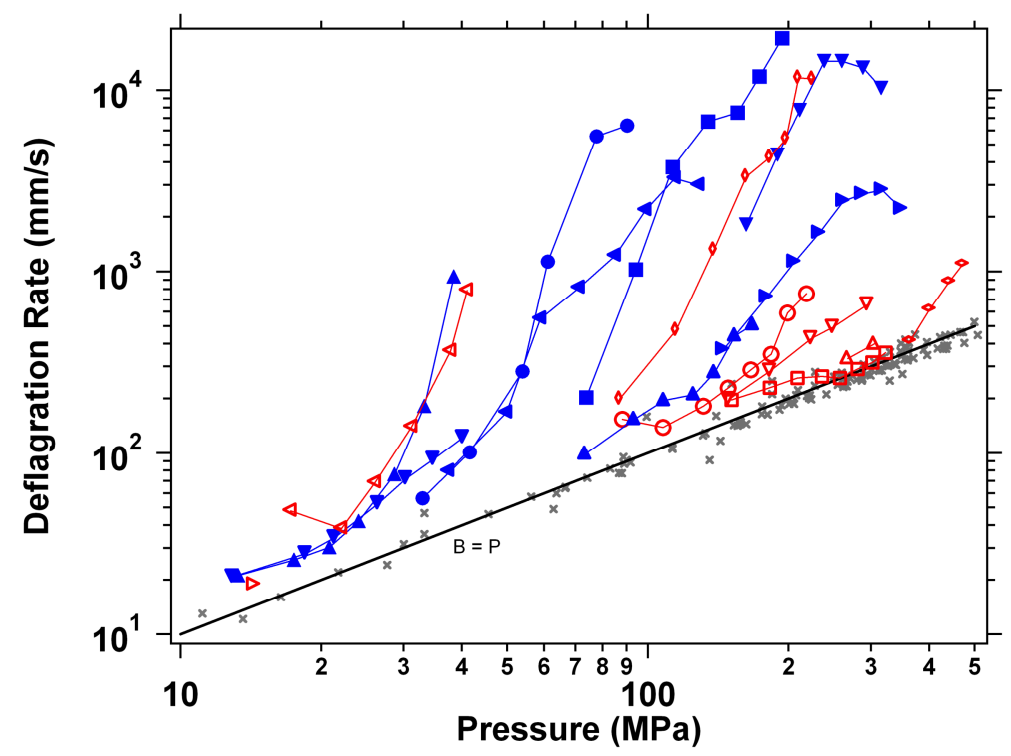

FIGURE 1. EDC-37 deflagration rates at ambient temperature are shown. Open symbols represent data collected in 2004, while the closed symbols represent data from 2006. Gray " $x$ "s represent LX-04 data and are included for comparison. The solid line represents the linear equation \{deflagration rate $\}=$ \{pressure , which fits LX-04 deflagration rate data

Nearly all energetic materials studied exhibit deflagration rates that increase with pressure. EDC-37 seems to be no exception. However, unlike many other materials studied, EDC-37 deflagration rates seem to be time dependent. That is, deflagration rates increase with the time of each experiment. For example, at one particular pressure, the measured deflagration rate for an experiment near the end of the run is faster than the deflagration rate for an experiment near the beginning of a run.

Most importantly, however, is the observation that EDC-37 deflagrates faster than other similar HMX-based formulations like LX-04. Consequently, the violence of thermal explosion may be higher; however, because the deflagration rate is only one indicator of the eventual violence of thermal explosion, further testing with apparatuses that measure thermal explosion violence directly could be used to provide additional insight into this hypothesis.

\section{REFERENCES}

1. J.L. Maienschein, J.F. Wardell, M.R. DeHaven, C.K. Black, "Deflagration of HMX-Based Explosives at High Temperatures and Pressures", in Propellants, Explosives, and Pyrotechnics 29, No. 5. (2004) 